\title{
Construction of novel conditional transgenic vectors for molecular therapy based on the Tet-On system and flanked with insulators in a single plasmid
}

\author{
YAN AN WANG ${ }^{1 *}$, ZHAO LIANG FEI ${ }^{2 *}$, ZHI YUAN ZHANG ${ }^{1}$, XIN QUAN JIANG ${ }^{1}$, \\ WAN TAO CHEN ${ }^{1}$, ZHU GANG WANG ${ }^{2}$, JIAN FEI ${ }^{2}$ and JIA WEI ZHENG ${ }^{1}$ \\ ${ }^{1}$ Department of Oral and Maxillofacial Surgery, Ninth People's Hospital, \\ Shanghai JiaoTong University School of Medicine, Shanghai Key Laboratory of Stomatology, Shanghai 200011; \\ ${ }^{2}$ Shanghai Research Center for Biomodel Organisms, Shanghai 201103, P.R. China
}

Received March 30, 2009; Accepted June 12, 2009

DOI: $10.3892 / \mathrm{mmr}$ _00000175

\begin{abstract}
Regulated expression of a gene of interest is crucial for transgenic research, as well as for safe and efficacious gene therapy. The most commonly used conditional expression system requires the generation of two transgenic strains, one carrying an inducible promoter and the other a transactivator. The generation of conditional transgenic models using this method is costly and time consuming. In this study, we report the design and construction of novel simplified gene delivery vectors that integrate both the regulatory and responsive elements in a single vector. The Tet-On system was used and integrated the inducible promoter and transactivator in a single plasmid, between which a copy of an insulator was inserted to minimize interference between the two units. Another copy of an insulator was inserted upstream of the transgene cassette to eliminate transgene silencing and to lower basal expression. The two insulators were in the same orientation. To further decrease basal expression, the most powerful repressor domain containing a 'kruppel-associated box' of the zinc finger protein NK10 was used and fused to TetR in one of the two vectors. The function of this system was confirmed after in vitro transient transfection. The two conditional plasmids were successfully constructed, and the expression of the gene of interest was regulated tightly in vitro. In conclusion, the vectors described here may be useful for gene therapy applications, as well as for the establishment of conditional animal models.
\end{abstract}

Correspondence to: Dr Jia Wei Zheng, Department of Oral and Maxillofacial Surgery, Ninth People's Hospital, Shanghai JiaoTong University School of Medicine, Shanghai Key Laboratory of Stomatology, Shanghai 200011, P.R. China

E-mail: zhjw@omschina.org.cn

*Contributed equally

Key words: conditional transgenics, transgene silencing, insulator, Tet-On system, gene therapy, plasmid construction

\section{Introduction}

Over the last two decades, fundamental insight into the function of genes has been efficiently provided through conventional knockout and transgenic experiments. Although genetically modified mice have provided important new information about the function of many genes, there are serious limitations to current animal models for a number of diseases. Introducing genetic changes to the germ line of a mouse may track down the effects of a particular gene, but may also have severe developmental consequences, complicating or even precluding the desired experimental analysis. A good example illustrating these restrictions is embryonic lethality. In gene therapy, an ideal vector should include a regulatory system that is off in the resting state, exhibit tight regulation, and allow for rapid and repeatable induction in response to a clinically approved inducer molecule. To overcome these undesired limitations and to precisely control gene expression, conditional mouse models are becoming increasingly popular. Although there are many types of inducible transgenic expression systems, tetracycline-controlled expression systems have been employed most frequently. The tet system, developed by Gossen and co-workers, includes two basic variants: the Tet-Off system (tTA) and the Tet-On system (rtTA) $(1,2)$. As a positive regulatory system, the Tet-On system has been used more widely in numerous transgenic animal models $(3,4)$. The original tet system contained two transcriptional units, the transactivator and the responder, on two plasmids. Currently available tetracycline-regulated transgenic methods require the generation of two transgenic strains, one carrying the transgene of interest under the control of the tet operator, and the other the reverse transactivator (rtTA). Crossing the lines generates progeny with both transgenes, allowing the regulation of the gene of interest through the administration of tetracycline. However, crossing and analysis of animals transgenic for the individual components of the system is costly and time consuming. Therefore, the generation of a vector containing the two units in a single plasmid may provide advantages for the simplified generation of conditional transgenic animals. The successful use of a single construct containing the two elements has been 
reported (5-7). In addition, improvements to newly created tetbased systems should include the elimination of background expression, increased sensitivity to tetracycline or its derivative, doxycyline (Dox), and increased inducibility.

In the present study, we designed and constructed two novel conditional transgenic vectors combining the two transcriptional units on a single plasmid. In addition, in order to eliminate the background expression of the gene of interest, the most powerful repressor domain containing a 'kruppelassociated box' of the zinc finger protein $\operatorname{NK} 10(8,9)$ was used and fused to tetR. The transactivator was placed under the control of the CMV promoter and the tet responsive element, driving the gene of interest, was inserted downstream of the same vector. To minimize any potential interference between the two elements, they were spaced with a $1.2-\mathrm{kb}$ cHS4 insulator. To shield the transgene from chromosomal position effects and to improve transgene expression efficiency, another copy of the cHS4 insulator was inserted upstream of the transgene cassette. The two insulators were placed in the same orientation.

\section{Materials and methods}

\section{Plasmid construction}

Construction of regulatory units. The first step of the study involved the cloning of the cHS4 insulator from chicken genomic DNA. The primers used were forward primer 5'AA AACGCCTCCAGGAAACCACTCT3' and reverse primer 5' CAGCAATATTCCCCCATCCTCACT3'. To ensure that the sequence was in frame with the targeting sequence, the pfu enzyme was used for the PCR experiments. PCR conditions were $95^{\circ} \mathrm{C}$ for $5 \mathrm{~min}$ followed by 34 cycles at $94^{\circ} \mathrm{C}$ for $30 \mathrm{sec}$, $60.1^{\circ} \mathrm{C}$ for $40 \mathrm{sec}$ and $72^{\circ} \mathrm{C}$ for $2 \mathrm{~min} 40 \mathrm{sec}$, and then $72^{\circ} \mathrm{C}$ for $10 \mathrm{~min}$.

The NK10 gene was cloned from brain tissue cDNA. The primers used were forward primer 5'GAATTCGGAATGGC CCCGAGACCT3' and reverse primer 5'CTGCAGTCAATGC CCCTGGATAGCGACATT 3'. EcoR1 and Pst I restriction site sequences were respectively incorporated into the $5^{\prime}$ end of the PCR primers. PCR conditions were $95^{\circ} \mathrm{C}$ for $5 \mathrm{~min}$ followed by 34 cycles at $94^{\circ} \mathrm{C}$ for $30 \mathrm{sec}, 61^{\circ} \mathrm{C}$ for $40 \mathrm{sec}$ and $72^{\circ} \mathrm{C}$ for $1 \mathrm{~min}$, and then $72^{\circ} \mathrm{C}$ for $10 \mathrm{~min}$.

The tetR gene was cloned from the plasmid of Ptet-off. The primers used were forward primer 5'AAACTCGAGCCCGGG ATGGCTAGATTAGATAAAAG 3' and reverse primer 5'AAA GAATTCCTTTCTCTTCTTTTTTGGCGACCCACTTTCA CATTT3'. The XhoI and EcoRI restriction site sequences were respectively introduced into the $5^{\prime}$ end of the PCR primers. PCR conditions were $95^{\circ} \mathrm{C}$ for 5 min followed by 34 cycles at $94^{\circ} \mathrm{C}$ for $30 \mathrm{sec}, 61.5^{\circ} \mathrm{C}$ for $40 \mathrm{sec}$ and $72^{\circ} \mathrm{C}$ for $1 \mathrm{~min}$, and then $72^{\circ} \mathrm{C}$ for $10 \mathrm{~min}$.

The 1.36-kb cHS4 insulator was digested by SacII and $S s p I$, and the $S a c I I-S s p$ I fragment $(1.2 \mathrm{~kb})$ was subcloned into the $S a c$ II-EcoRV fragment of plasmid pSK- to yield the plasmid pBSHs4. The NK10 and tetR PCR products were respectively subcloned into the T-vector. The pMD-NK10 plasmid was digested by PstI and EcoR1, and pMD-tetR was digested by XhoI and EcoRI. The PstI-EcoRI fragment, containing the NK10 gene, and the XhoI-EcoRI fragment, containing the tetR gene, were subcloned into the PstI-XhoI fragment of the $\mathrm{pSK}^{-}$ plasmid to yield the plasmid pBSNK10-tetR, in which the NK10-tetR gene could be translated into a fusion protein. The plasmid pBSNK10-tetR was digested by XhoI. After the end was blunted and purified, the fragment was digested by Not I. The plasmid pIRES-EGFP was digested by BstXI. After the end was blunted and purified, the fragment was also digested by NotI, cutting out the EGFP gene. The XhoI-NotI insert fragment containing the NK10-tetR gene and the BstXI-NotI vector fragment were ligated together to generate the plasmid pIRES-NK10-tetR. To build the plasmid pVP16-NK10-tetR, an $E c o$ RI-Pst I digestion was carried out on the plasmid pIRESNK10-tetR, generating the 4-kb EcoRI-PstI vector fragment. A BamH1-Pst 1 double digestion was also performed on the same plasmid to obtain the 1.6-kb BamH1-Pst 1 insert fragment. The ptet-on plasmid was digested by EcoR1 and BamHI, isolating the 1.6-kb EcoRI-BamHI insert fragment rtTA. The three fragments, EcoRI-PstI vector fragment, BamHI-Pst I insert fragment and EcoRI-BamHI insert fragment, which all had sticky ends, were ligated in one ligation reaction, and the pVP16-NK10-tetR plasmid was constructed. The pVP16NK10-tetR plasmid was digested with BamHI and NotI, cutting out the IRES-NK10-tetR fragment, and was then itself ligated after its end was blunted, generating the plasmid pVsp-teton.

\section{Construction of responsive elements}

Cloning of the MT gene. The plasmid pPyMT1 containing the MT gene was digested by BstXI restriction enzyme. A $0.8 \%$ agarose gel electrophoresis loaded with all the samples was used to isolate the $1.53-\mathrm{kb}$ fragments. After the ends were blunted and purified, the fragments were digested by HindIII, and the BstXI (blunting)-HindIII fragment (1.48 kb) containing an open reading frame of the MT gene was subcloned into the SmaI-HindIII fragment of pSK- yielding the plasmid PbsMT. A BamHI-HindIII digestion reaction was carried out on the plasmid PbsMT, and the BamHI-HindIII fragment containing the MT gene was cloned to the BamHI-HindIII vector fragment of the pTRE2 plasmid, yielding the vector pTREMT.

The pBSHs 4 plasmid was first digested with ClaI, and then SacI digestion was carried out on the same fragment after the ClaI ends were blunted. The 1.2-kb ClaI (blunt)-SacI insert fragment containing the Hs4 insulator was ligated with the SacI-EcoRI vector fragment of the pN2-EGFP plasmid for which the EcoRI ends were also blunted. This plasmid was labeled pHs4-N2EGFP. The XhoI-BamHI fragment of the pTRE2 plasmid containing the TRE gene was cloned to the Sal1-BamH1 vector fragment of the pHs4-N2EGFP plasmid. As $\mathrm{XhoI}$ is an isoschizomer of SalI, this ligation reaction was sticky-ended, and the plasmid was named pHs4TRE-N2EGFP. The next step was to replace the EGFP gene of the pHs4TREN2EGFP vector with MT. The experiments were carried out as follows: pHs4TRE-N2EGFP was digested with BamHI and NotI, the NotI ends were blunted, and the vector fragment was ligated with the BamHI-EcoRV insert fragment of the pTRE-MT plasmid containing the MT gene. This plasmid was named pN2-Hs4TRE-MT.

Ligation of regulatory units and responsive elements. The SspI-VspI insert fragment of the pVP16-NK10-tetR plasmid $(2.5 \mathrm{~kb})$ and pVsp-teton $(1.9 \mathrm{~kb})$ were respectively ligated with the NheI- VspI vector fragment of pN2-Hs4TRE-MT, whose 
NheI ends were blunted. Thus, the plasmids pVP16NK10-Hs4TREMT and pVP16-Hs4-TREMT were constructed.

In order to insert another Hs4 insulator upstream of the transgene cassette, a plasmid was constructed. First, a pair of primers was designed: forward primer 5'AAAATCGATCATA TGGCGGAGCGAGGAACTGAG3', having ClaI and NdeI restriction site sequences, and reverse primer 5'AAACTC GAGGATATCCATATGCCGCCCTGGGAA3,' having XhoI, $E c o$ RV and NdeI restriction site sequences. The template DNA was the 1.5-kb MT gene, originated from the PbsMT plasmid. PCR was carried out to amplify a 500-bp fragment. The product as a spacer was digested with Cla 1 and Xho1 and cloned to the Cla1-XhoI vector fragment of pBSHs4. Thus, the pBSHs4-Nde plasmid was constructed. Upstream of the spacer was the NdeI restriction site, and downstream were $N d e \mathrm{I}$ and $E c o$ V. Therefore, the VspI-SspI insert fragments of pVP16NK10-Hs4-TREMT $(6.9 \mathrm{~kb})$ and pVP16-Hs4-TREMT $(5.4 \mathrm{~kb})$ could theoretically be cloned to the pBSHs4-Nde's NdeI-EcoRV vector fragment (4.2 kb). However, despite numerous attempts, we did not succeed in acheiving this, and thus changed our strategy. The pBSHs4-Nde plasmid was first digested with Vsp1 and Nde1. The 1.4-kb fragment containing two types of DNAs was extracted, and Ssp 1 digestion was carried out. Thus, two types of DNAs were divided, and the 1.4-kb fragment containing the $1.2-\mathrm{kb}$ insulator flanking the sticky ends of VspI and NdeI was respectively cloned to the VspI site of the plasmids pVP16NK10-Hs4-TREMT and pVP16-Hs4-TREMT, yielding the final plasmids pHs4-VP16NK10-Hs4-TREMT and pHs4VP16-Hs4-TREMT. BstX1 digestion was performed to determine the insert directions.

In vitro experiments with the two plasmids. To confirm the function of the two plasmids, transient transfection was carried out. COS7 cells were cultivated in DMEM for $72 \mathrm{~h}$ and transfected by electroporation with the same amount of the two plasmids. The electroporation conditions were: voltage, $220 \mathrm{~V}$; electrical capacity, $975 \mu \mathrm{F}$; no. of pulses, 1 . After electroporation and the addition of $1.5 \mathrm{ml}$ DMEM to the cuvette, equal amounts of cells were carefully transferred to two culture dishes. Dox (15 $\mu \mathrm{g} / \mathrm{ml}$ DMEM) was added to one dish $3 \mathrm{~h}$ after electroporation, and the other dish was used as the control.

Semi-quantitative reverse transcription-polymerase chain reaction (RT-PCR) was applied to measure the marker gene expression of the two plasmids. After $72 \mathrm{~h}$ of incubation, total RNA was isolated from the cells by the TRIzol method according to the manufacturer's instructions (Invitrogen, Cergy-Pontoise, France). In order to resolve the problem of genomic DNA contamination, $2 \mu \mathrm{g}$ RNA was digested with RNase Free DNase I (New England BioLab, UK). RT-PCR was carried out with $1 \mu \mathrm{g}$ of DNase-digested RNA. cDNA was generated using the First-Strand Synthesis System for RT-PCR (Takara, Dalian) followed by PCR amplification of the marker gene. Expression of $\beta$-actin, a housekeeping gene which is assumed to be expressed at roughly equal levels in different cell types and under different conditions, was examined as an internal control. The primers and templates used the same amount of substances in different reactions. The primers used were the MT forward primer 5'AAAGAATTCCCATTTTTT GGCTGGGCAGATATCGCGGAGCGAGGAACTGAG3' and MT reverse primer 5'AAAAAGCTTCCATTTTTTGGAT

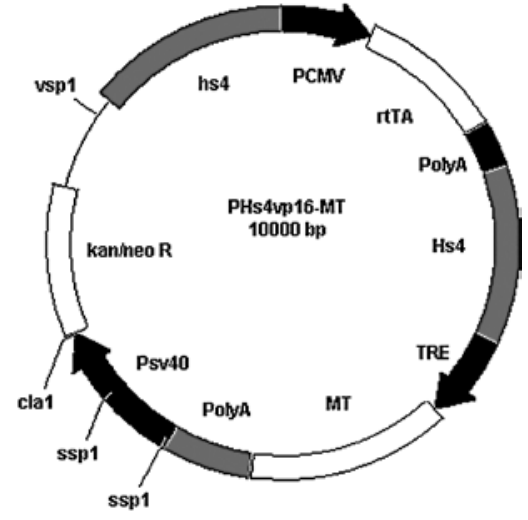

Figure 1. Map of the plasmid pHs4vp16-MT.

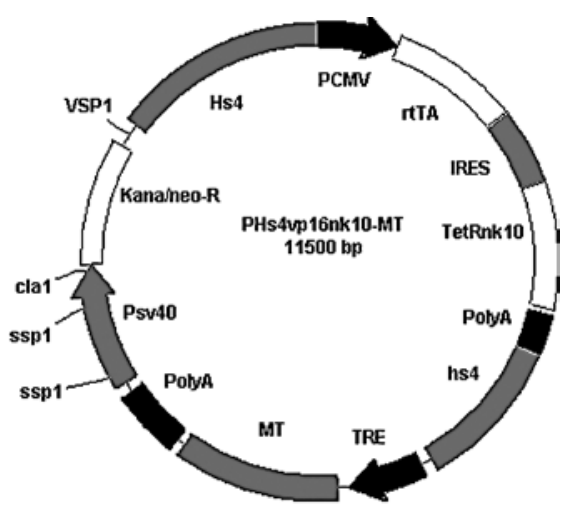

Figure 2. Map of the plasmid pHs4vp16nk10-MT.

GGCGAGATATCCGCCCTGGGAATGATAG3'. $\beta$-actin PCR primers were kindly provided by Dr Fei (IBCB, Shanghai Institutes for Biological Sciences, Chinese Academy of Sciences) and were designed to span an intron of the genomic sequence, resulting in a PCR product from genomic contamination that was larger in size than the product generated from the cDNA. The PCR conditions were $95^{\circ} \mathrm{C}$ for $5 \mathrm{~min}$ followed by 27 cycles at $94^{\circ} \mathrm{C}$ for $30 \mathrm{sec}, 60^{\circ} \mathrm{C}$ for $1 \mathrm{~min} 30 \mathrm{sec}$ and $72^{\circ} \mathrm{C}$ for $1 \mathrm{~min}$, and then $72^{\circ} \mathrm{C}$ for $10 \mathrm{~min}$. Agarose gel (0.8\%) loaded with the same volume of PCR products was run to test the differences in marker gene expression levels.

\section{Results}

For the construction of the vectors, the following plasmids were used: pTet-On, pTet-Off, pTRE2, PBSsk, pIRES2-EGFP and pEGFP-N2, and the backbone of the final plasmid was pEGFP-N2. The Tet-Off and Tet-On systems were developed to regulate transgene expression, respectively providing negative and positive control of transgene expression. Although the Tet-On system produces high basal expression in the absence of an inducer, as a positive regulation system it is widely applied. In order to eliminate the basal expression, we cloned the 'kruppel-associated box' silencing domain of the zinc finger protein NK10 gene, a powerful transcriptional repressor, and ligated it with tet repressor to translate a fusion protein. The fusion pro- 


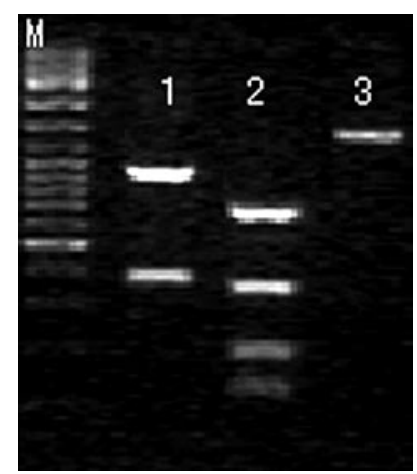

Figure 3. cHS4 insulator restriction enzyme digestion. Lane M, DNA ladder; 1, HindIII digestion; 2, PstI digestion; 3, cHS4 PCR products.
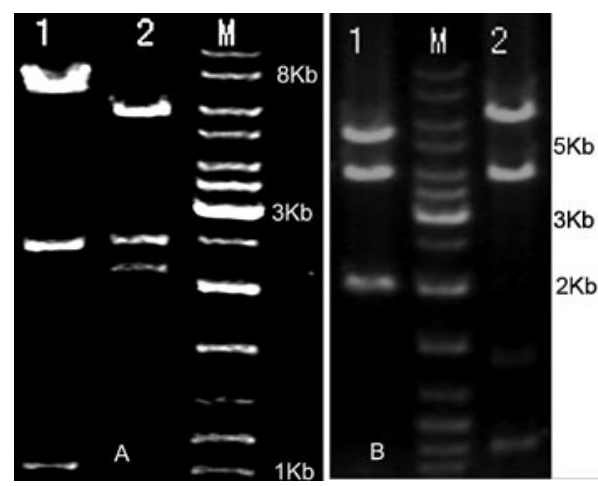

Figure 4. Results of BstxI digestion for the plasmids pHs4vp16-MT (A) and pHs4vp16nk10-MT (B). (A) Lane 1, positive cloning; 2, negative cloning; M, DNA ladder. (B) Lane 1, negative cloning; 2, positive cloning; M, DNA ladder.

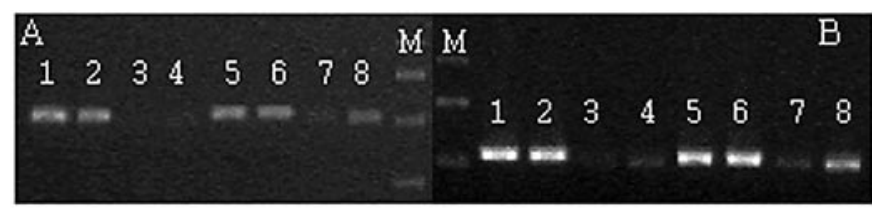

Figure 5. The results of semi-quantitative RT-PCR of the plasmids pHs4vp16nk10-MT and pHs4vp16-MT (lane 7 and 8). Cycles 22 (A) and 24 (B) are shown. No Dox was added in lanes 1, 3, 5 or 7; Dox was added in lanes 2, 4, 6 and 8 . No difference was observed in $\beta$-actin expression before and after the addition of Dox (lanes 1, 2, 5 and 6). M, DNA ladder.

tein binds to the tet operator sequences in the response element only in the absence of Dox, and reduces leaked gene transcription. When Dox is added, the silencer dissociates from the tet operator, relieving transcriptional inhibition, while rtTA binds to the TRE and activates gene expression. To compare the efficiency and effectiveness of diverse operating systems, two different Tet-On-based inducible expression systems carried in a single cassette were constructed (Figs. 1 and 2).

In this study, three genes were successfully cloned using the PCR method. The sequences were verified using sequencespecific identification and restriction enzyme digestion (Fig. 3).

Restriction digestions were carried out to identify the final two transgene vectors, and analysis of the gel showed that the two plasmids were successfully constructed (Fig. 4).
The relative quantification of MT target transcripts was conducted using semi-quantitative RT-PCR in mRNA samples by comparison with the mRNA abundance of $\beta$-actin. The results showed that the plasmids pHs4vp16nk10-MT and pHs4vp16-MT had certain levels of transgene expression in the absence of tetracycline. The inducibility of pHs4vp16-MT was higher than that of pHs4vp16nk10-MT (Fig. 5).

\section{Discussion}

Transgenic models have provided a wealth of information regarding the function of specific genes. However, the limitations of promoter-controlled transgene expression have become apparent. The constitutive system has no control over the time of expression, which depends entirely on the properties of the promoters used. The promoters in this setting were constitutively active, many from early in the embryonic stages. The target gene is activated as soon as the promoter becomes active. If the transgene product happens to be toxic to the organism, particularly during gestation, it can be detrimental to the developing embryo. This results in the failure to generate live progeny carrying the transgene, and the transgene cannot be studied past embryogenesis. If the gene product is not toxic to the embryos, then the animals are viable and may demonstrate an interesting phenotype. However, it is uncertain whether the phenotype is the result of transgene expression or the mutation of endogenous genes. To address these limitations, a number of investigators have established transgenic systems in which the expression of the transgene can be externally regulated. Although a variety of approaches have been utilized, tetracycline-controlled expression systems have been employed most frequently (10).

In the present study, we successfully constructed a single plasmid containing a tet-responsive transactivator together with the transgene responder. This strategy circumvents the need to generate two independent transgenetic lines, and greatly facilitates mouse breeding as the effector and responder will not segregate.

A recent development in gene transfer approaches in plants and animals has revealed that transgenes undergo silencing after integration in the genome (11-13). Gene expression is influenced not only by the presence or absence of nearby transcription factors, but also by the structure of surrounding chromatin. Variable repression of transcription is often associated with heterochromatin and frequently manifests itself as position effect variegation. In an extensive study of this phenomenon, Cerutti et al (12) concluded that in C. reinhardtii the local heterochromatin structure rather than DNA methylation has a role in transgene inactivation. Insulators are DNA sequence elements that function to block the action of an enhancer and shield a transgene from a chromosomal position effect in transgenic animals and cell lines (14). Flanking a transgene with two copies of an insulator can shield it from a chromosomal position effect following stable integration. In a preliminary study, we attempted to insert two copies of 1.2-kb insulators into the flank of the transgene cassette (three copies of direct repeat sequences in one plasmid might cause it to be unstable). Despite numberous attempts, we did not succeed, even though STBL4 competent cells capable of stabilizing direct repeat sequences were used. Transgenes are usually 
found inserted into a single chromosomal location as multiple copies forming head-to-tail concatermers (10), while insulators can be effective when placed to only one side of the transgenes in multicopy arrays. This strategy was successfully employed in this study and others (15-17). Theoretically, only the end copy of the transgene is susceptible to a position effect after the concatemers are integrated with germ line genomic DNA. This not only reduces cloning time and decreases plasmid size, but also improves transgene expression efficiency.

Tetracycline-inducible transgene expression systems should have negligible basal expression levels and high inducibility. However, these goals were not acheived in the present study. Background expression remained in the two vectors, though the background expression in pHs4vp16nk10-MT was much lower than that of pHs4vp16-MT, while the inducibility of the pHs4vp16-MT was much higher than that of pHs4vp16nk10 -MT. The reasons are as follows: first, there was an enhancer/ promoter of the neo gene in the two plasmids, which also acts on the CMV minipromoter of TR E, promoting the expression of the target gene from a distance. In in vivo experiments, this factor will be excluded after the linearization of the plasmid with VspI and SspI digestions. Second, in the plasmid of pHs4vp16nk10-MT, the products rtTA and tetR-NK10 can form heterodimers that do not function appropriately and compromise the overall inducibility of this system. On the other hand, competition exists between rtTA and tetR-NK10 for the DNA binding sites of TRE, which also lowers Dox inducibility. Basal expression was also associated with the site(s) of integration of the transgenic constructs, and enhancer sequences near the target gene have been repeatedly demonstrated to increase basal target gene expression, thereby compromising the desired tight regulation $(18,19)$. To eliminate basal expression in our study, an insulator was used to block the effect of the enhancer near the transgene integration sites on TRE. Although the results of transient transfection testing showed a degree of leakage expression, in some cases the leak that resulted was acceptable and did not negate the ability of the experimental system to appropriately address the investigated hypothesis. Upstream of each of our transgene cassettes was a neo gene. When the vectors were only digested with $S s p$ I, this gene, being a selective marker, was included in the transgene cassette. This benefited the stable transfection of the two vectors in vitro.

In summary, we combined two expression units of the Tet-On system on a single plasmid by constructing two conditional transgenic vectors, one of which contained the NK10 gene fused to TetR. cHS4 insulator elements were utilized in the construction of the vectors to eliminate basal expression and transgene silencing. Our in vitro study demonstrated that the two 'two-in-one' systems were functional. This may minimize the breeding and genotyping time required for phenotypic characterization in the construction of conditional animal models. Moreover, when the vectors were digested only with $S s p$ I, a neo gene as a selection marker was included in the transgene cassettes. Therefore, the Tet-On system presented here should be easy to use and widely applicable to the study of gene function in transfected cells and molecular therapy, as well as for the creation of conditional transgenic animal models to study disease and development in vivo.

\section{Acknowledgements}

This work was supported by the National Natural Science Foundation of China (grant no. 30371546), the Technology Commission of Shanghai (no. 08DZ2271100) and the Shanghai Leading Academic Discipline Project (project no. S30206).

\section{References}

1. Gossen M and Bujard H: Tight control of gene expression in mammalian cells by tetracycline-responsive promoters. Proc Natl Acad Sci USA 89: 5547-5551, 1992.

2. Gossen M, Freundlieb S, Bender G, Muller G, Hillen W and Bujard $\mathrm{H}$ : Transcriptional activation by tetracyclines in mammalian cells. Science 268: 1766-1769, 1995.

3. Vitale-Cross L, Amornphimoltham P, Fisher G, Molinolo AA and Gutkind JS: Conditional expression of K-ras in an epithelial compartment that includes the stem cells is sufficient to promote squamous cell carcinogenesis. Cancer Res 64: 8804-8807, 2004.

4. Zabala M, Wang L, Hernandez-Alcoceba R, et al: Optimization of the Tet-on system to regulate interleukin 12 expression in the liver for the treatment of hepatic tumors. Cancer Res 64: 2799-2804, 2004.

5. Lottmann H, Vanselow J, Hessabi B and Walther R: The Tet-On system in transgenic mice: inhibition of the mouse pdx-1 gene activity by antisense RNA expression in pancreatic beta-cells. J Mol Med 79: 321-328, 2001

6. Backman CM, Zhang Y, Hoffer BJ and Tomac AC: Tetracyclineinducible expression systems for the generation of transgenic animals: a comparison of various inducible systems carried in a single vector. J Neurosci Methods 139: 257-262, 2004.

7. Mizuguchi H, Xu ZL, Sakurai F, Mayumi T and Hayakawa T: Tight positive regulation of transgene expression by a single adenovirus vector containing the rtTA and tTS expression cassettes in separate genome regions. Hum Gene Ther 14: 1265-1277, 2003.

8. Thiel G, Lietz M, Bach K, Guethlein L and Cibelli G: Biological activity of mammalian transcriptional repressors. Biol Chem 382 : 891-902, 2001

9. Lange R, Christoph A, Thiesen HJ, et al: Developmentally regulated mouse gene NK10 encodes a zinc finger repressor protein with differential DNA-binding domains. DNA Cell Biol 14: 971-981, 1995.

10. Zhu Z, Ma B, Homer RJ, Zheng $\mathrm{T}$ and Elias JA: Use of the tetracycline-controlled transcriptional silencer (tTS) to eliminate transgene leak in inducible overexpression transgenic mice. J Biol Chem 276: 25222-25229, 2001.

11. Ingelbrecht I, van Houdt H, van Montagu M and Depicker A: Posttranscriptional silencing of reporter transgenes in tobacco correlates with DNA methylation. Proc Natl Acad Sci USA 91: 10502-10506, 1994.

12. Cerutti H, Johnson AM, Gillham NW and Boynton JE: Epigenetic silencing of a foreign gene in nuclear transformants of Chlamydomonas. Plant Cell 9: 925-945, 1997.

13. Finnegan EJ, Genger RK, Peacock WJ and Dennis ES: DNA methylation in plants. Annu Rev Plant Physiol Plant Mol Biol 49: 223-247, 1998.

14. Burgess-Beusse B, Farrell C, Gaszner M, et al: The insulation of genes from external enhancers and silencing chromatin. Proc Natl Acad Sci USA 99 (suppl 4): 16433-16437, 2002.

15. Boeda B, Weil D and Petit C: A specific promoter of the sensory cells of the inner ear defined by transgenesis. Hum Mol Genet 10: 1581-1589, 2001.

16. Rivella S, Callegari JA, May C, Tan CW and Sadelain M: The cHS4 insulator increases the probability of retroviral expression at random chromosomal integration sites. J Virol 74: 4679-4687, 2000.

17. Taboit-Dameron F, Malassagne B, Viglietta C, et al: Association of the 5'HS4 sequence of the chicken beta-globin locus control region with human EF1 alpha gene promoter induces ubiquitous and high expression of human CD55 and CD59 cDNAs in transgenic rabbits. Transgenic Res 8: 223-235, 1999.

18. Furth PA, St Onge L, Boger H, et al: Temporal control of gene expression in transgenic mice by a tetracycline-responsive promoter. Proc Natl Acad Sci USA 91: 9302-9306, 1994.

19. Zheng T, Zhu Z, Wang Z, Homer RJ and Ma B: Inducible targeting of IL-13 to the adult lung causes matrix metalloproteinase- and cathepsin-dependent emphysema. J Clin Invest 106: 1081-1093, 2000. 